\title{
Finite speed of propagation for the thin film equation in spherical geometry
}

\author{
Roman M. Taranets \\ Institute of Applied Mathematics and Mechanics of the NASU, \\ Sloviansk, Ukraine, taranets_r@yahoo.com
}

August 13, 2018

\begin{abstract}
We show that a double degenerate thin film equation, which originated from modeling of viscous coating flow on a spherical surface, has finite speed of propagation for nonnegative strong solutions and hence there exists an interface or free boundary separating the regions where solution $u>0$ and $u=0$. Using local entropy estimates we also obtain an upper bound for the rate of the interface propagation.
\end{abstract}

UDC 517.953

MSC2010: 35K 35, 35K55, 35K65, 35B45, 35B65

\section{Introduction}

In this paper, we study a particular case of the following doubly degenerate fourth-order parabolic equation

$$
u_{t}+\left[u^{n}\left(1-x^{2}\right)\left(a-b x+c\left(2 u+\left(\left(1-x^{2}\right) u_{x}\right)_{x}\right)_{x}\right)\right]_{x}=0 \text { in } Q_{T},
$$

where $u(x, t)$ represents the thickness of the thin film, the dimension-

less parameters $a, b$ and $c$ describe the effects of gravity, rotation and 
surface tension, $Q_{T}=\Omega \times(0, T), n>0, T>0$, and $\Omega=(-1,1)$. For $n=3$ (no-slip regime) this equation describes the dynamics of a thin viscous liquid film on the outer surface of a solid sphere. For $n=2$ the classical Navier slip condition is recovered. On the other hand, parameter ranges $n \in(0,2)(n \in(2,3))$ in the equation (1.1) correspond to strong (weak) wetting slip regimes. More general dynamics of the liquid film for the case when the draining of the film due to gravity was balanced by centrifugal forces arising from the rotation of the sphere about a vertical axis and by capillary forces due to surface tension was considered in [11]. In addition, Marangoni effects due to temperature gradients were taken into account in [12]. The spherical model without the surface tension and Marangoni effects was studied in [17, 18].

We are interested in time evolution of the support of non-negative strong solutions to

$$
u_{t}+\left(\left(1-x^{2}\right)|u|^{n}\left(\left(1-x^{2}\right) u_{x}\right)_{x x}\right)_{x}=0 .
$$

Equation (1.2) is a particular case of (1.1) with $a=b=0$ with an absence of the second-order diffusion term. Existence of weak solutions for (1.2) in a weighted Sobolev space was shown in [13] and existence of more regular non-negative strong solutions of (1.2) was recently proved in [16. Unlike the classical thin film equation

$$
u_{t}+\left(|u|^{n} u_{x x x}\right)_{x}=0
$$

the qualitative behavior of solutions for double degenerate thin-film equation (1.2) is still not well understood. Note that the model equation (1.3) describes the coating flow of a thin viscous film on a flat surface under the surface tension effect. Depending on the value of the parameter $n$ non-negative solutions of this equation posses some properties. For example, in 1990, Bernis and Friedman [2] defined and constructed non-negative weak solutions of the equation (1.3) when $n \geqslant 1$, and it was also shown that for $n \geqslant 4$, with a uniformly positive initial condition, there exists a unique positive classical solution. Later on, in 1994, Bertozzi et al. 6] generalised this positivity property for the case $n \geqslant \frac{7}{2}$. In 1995, Beretta et al. [1] proved the existence of 
non-negative weak solutions for the equation (1.3) if $n>0$, and the existence of strong ones for $0<n<3$. Also, they could show that this positivity-preserving property holds at almost every time $t$ in the case $n \geqslant 2$. This positivity-preservation result was generalized for a cylindrical surface was obtained in [7]. Furthermore, for $n \geqslant \frac{3}{2}$ the solution's support to (1.3) is non-decreasing in time, and the support remains constant if $n \geqslant 4$. The existence (nonexistence) of compactly supported spreading source type solution to (1.3) was demonstrated for $0<n<3(n \geqslant 3)$ in [5]. One of interesting qualitative properties of non-linear parabolic thin film equations is finite speed of support propagation that is not the case when the parabolic equation is a linear one. This property was first shown in [3] if $0<n<2$, and in [4, 10] if $2 \leqslant n<3$ for non-negative strong solutions of (1.3). A similar result on a cylindrical surface was obtained in [8].

Our main result for the thin film equation on the spherical surface is the finite speed of the interface propagation in the special case of the strong slip regime $n \in(1,2)$. Proof of the finite speed of propagation property is based on local entropy estimate and Stampacchia's lemma. Moreover, we obtain an upper bound the time evolution of the

support as: $\Gamma(t) \leqslant C_{0} t^{\frac{1}{n+4}}$. This bound coincides with the asymptotic behaviour of self-similar type solutions to (1.3) (see [5]).

\section{Main result}

We study the following thin film equation

$$
u_{t}+\left(\left(1-x^{2}\right)|u|^{n}\left(\left(1-x^{2}\right) u_{x}\right)_{x x}\right)_{x}=0 \text { in } Q_{T}
$$

with the no-flux boundary conditions

$$
\left(1-x^{2}\right) u_{x}=\left(1-x^{2}\right)\left(\left(1-x^{2}\right) u_{x}\right)_{x x}=0 \text { at } x= \pm 1, t>0,
$$

and the initial condition

$$
u(x, 0)=u_{0}(x) .
$$


Here $n>0, Q_{T}=\Omega \times(0, T), \Omega:=(-1,1)$, and $T>0$. Integrating the equation (2.1) by using boundary conditions (2.2), we obtain the mass conservation property

$$
\int_{\Omega} u(x, t) d x=\int_{\Omega} u_{0}(x) d x=: M>0 .
$$

Consider initial data $u_{0}(x) \geqslant 0$ for all $x \in \bar{\Omega}$ satisfying

$$
\int_{\Omega}\left\{u_{0}^{2}(x)+\left(1-x^{2}\right) u_{0, x}^{2}(x)\right\} d x<\infty .
$$

Definition 2.1. [weak solution/ Let $n>0$. A function $u$ is a weak solution of the problem (2.1)-(2.3) with initial data $u_{0}$ satisfying (2.5) if $u(x, t)$ has the following properties

$$
\begin{gathered}
\left(1-x^{2}\right)^{\frac{\beta}{2}} u \in C_{x, t}^{\frac{\alpha}{2}, \frac{\alpha}{8}}\left(\bar{Q}_{T}\right), 0<\alpha<\beta \leqslant \frac{2}{n}, \\
u_{t} \in L^{2}\left(0, T ;\left(H^{1}(\Omega)\right)^{*}\right),\left(1-x^{2}\right)^{\frac{1}{2}} u_{x} \in L^{\infty}\left(0, T ; L^{2}(\Omega)\right), \\
\left(1-x^{2}\right)^{\frac{1}{2}}|u|^{\frac{n}{2}}\left(\left(1-x^{2}\right) u_{x}\right)_{x x} \in L^{2}(P),
\end{gathered}
$$

u satisfies (2.1) in week sense:

$$
\int_{0}^{T}\left\langle u_{t}, \phi\right\rangle d t-\iint_{P}\left(1-x^{2}\right)|u|^{n}\left(\left(1-x^{2}\right) u_{x}\right)_{x x} \phi_{x} d x d t=0
$$

for all $\phi \in L^{2}\left(0, T ; H^{1}(\Omega)\right)$, where $P:=\bar{Q}_{T} \backslash\{\{u=0\} \cup\{t=0\}\}$,

$$
\left(1-x^{2}\right)^{\frac{1}{2}} u_{x}(., t) \rightarrow\left(1-x^{2}\right)^{\frac{1}{2}} u_{0, x}(.) \text { strongly in } L^{2}(\Omega) \text { as } t \rightarrow 0,
$$

and boundary conditions (2.2) hold at all points of the lateral boundary, where $\{u \neq 0\}$.

Let us denote by

$$
0 \leqslant G_{0}(z):=\left\{\begin{array}{l}
\frac{z^{2-n}-A^{2-n}}{(n-1)(n-2)}-\frac{A^{1-n}}{1-n}(z-A) \text { if } n \neq 1,2 \\
z \ln z-z(\ln A+1)+A \text { if } n=1 \\
\ln \left(\frac{A}{z}\right)+\frac{z}{A}-1 \text { if } n=2
\end{array}\right.
$$

where $A=0$ if $n \in(1,2)$ and $A>0$ if else. 
Theorem 1 (strong solution). Assume that $n \geqslant 1$ and initial data $u_{0}$ satisfies $\int_{\Omega} G_{0}\left(u_{0}\right) d x<+\infty$ then the problem (2.1)-(2.3) has a nonnegative weak solution, $u$, in the sense of Definition 2.1, such that

$$
\begin{gathered}
\left(1-x^{2}\right) u_{x} \in L^{2}\left(0, T ; H^{1}(\Omega)\right),\left(1-x^{2}\right)^{\frac{\gamma}{2}} u_{x} \in L^{2}\left(Q_{T}\right), \gamma \in(0,1], \\
u \in L^{\infty}\left(0, T ; L^{2}(\Omega)\right),\left(1-x^{2}\right)^{\frac{\mu}{2}} u \in L^{2}\left(Q_{T}\right), \quad \mu \in(-1, \beta] .
\end{gathered}
$$

The existence of these solutions was proved in [16]. Our aim is to establish the finite speed of propagation property for a strong solution $u$ of (2.1) in the sense of Theorem 1 .

Theorem 2 (finite speed of propagation). Assume that $1<n<2$, the initial data satisfies the hypotheses of Theorem 1 and the support of the initial data satisfies $\operatorname{supp}\left(h_{0}\right) \subset \Omega \backslash\left(-r_{0}, r_{0}\right)$, where $\Omega=(-1,1)$ and $r_{0} \in(0,1)$. Let $h$ be the strong solution from Theorem 1 . Then there exists a time $T^{*}>0$ and a nondecreasing function $\Gamma(t) \in C\left(\left[0, T^{*}\right]\right)$, $\Gamma(0)=0$ such that $u$ has finite speed propagation, $i . e$.

$$
\operatorname{supp}(u(\cdot, t)) \subseteq\left[-r_{0}+\Gamma(t), r_{0}-\Gamma(t)\right] \subset \Omega
$$

for all $t \in\left[0, T^{*}\right]$. Moreover, $\Gamma_{\text {opt }}(t)=C_{0} t^{\frac{1}{n+4}}$ for all $t \in\left[0, T^{*}\right]$.

\section{Proof of Theorem 2}

\subsection{Local entropy estimate}

Lemma 3.1. Assume that $1<n<2$ and $\nu>1$. Let $\zeta \in C_{t, x}^{1,2}\left(\bar{Q}_{T}\right)$ such that its support satisfies $\sup (\zeta) \subseteq \Omega$ and $\left(\zeta^{4}\right)_{x}=0$ on $\partial \Omega$. Then there exist positive constants $C_{1}, C_{2}$ are independent of $\Omega$, such that 
for all $T>0$ the strong solution $u$ of Theorem 1 satisfies

$$
\begin{gathered}
\int_{\Omega}\left(1-x^{2}\right)^{\nu} \zeta^{4}(x, T) G_{0}(u) d x-\iint_{Q_{T}}\left(1-x^{2}\right)^{\nu}\left(\zeta^{4}\right)_{t} G_{0}(u) d x d t+ \\
C_{1} \iint_{Q_{T}}\left(1-x^{2}\right)^{\nu+2} u_{x x}^{2} \zeta^{4} d x d t \leqslant \int_{\Omega}\left(1-x^{2}\right)^{\nu} \zeta^{4}(x, 0) G_{0}\left(u_{0}\right) d x \\
C_{2} \iint_{Q_{T}}\left(1-x^{2}\right)^{\nu} u_{x}^{2}\left[\zeta^{4}+\zeta^{2} \zeta_{x}^{2}+\zeta^{3}\left|\zeta_{x x}\right|\right] d x d t+ \\
C_{2} \iint_{Q_{T}}\left(1-x^{2}\right)^{\nu-2} u^{2}\left[\zeta^{4}+\zeta_{x}^{4}+\zeta^{2} \zeta_{x x}^{2}\right] d x d t
\end{gathered}
$$

Proof of Lemma 3.1. Equation (2.1) is doubly degenerate when $u=0$ and $x= \pm 1$. Therefore, for any $\epsilon>0$ and $\delta>0$ we consider twoparametric regularised equations

$$
u_{\epsilon \delta, t}+\left[\left(1-x^{2}+\delta\right)\left(\left|u_{\epsilon \delta}\right|^{n}+\epsilon\right)\left(\left(1-x^{2}+\delta\right) u_{\epsilon \delta, x}\right)_{x x}\right]_{x}=0 \text { in } Q_{T}
$$

with boundary conditions

$$
u_{\epsilon \delta, x}=\left(\left(1-x^{2}+\delta\right) u_{\epsilon \delta, x}\right)_{x x}=0 \text { at } x= \pm 1,
$$

and initial data

$$
u_{\epsilon \delta}(x, 0)=u_{0, \epsilon \delta}(x) \in C^{4+\gamma}(\bar{\Omega}), \gamma>0,
$$

where

$$
\begin{gathered}
u_{0, \epsilon \delta}(x) \geqslant u_{0 \delta}(x)+\epsilon^{\theta}, \quad \theta \in\left(0, \frac{1}{2(n-1)}\right), \\
u_{0, \epsilon \delta} \rightarrow u_{0 \delta} \text { strongly in } H^{1}(\Omega) \text { as } \epsilon \rightarrow 0, \\
\left(1-x^{2}+\delta\right)^{\frac{1}{2}} u_{0 x, \delta} \rightarrow\left(1-x^{2}\right)^{\frac{1}{2}} u_{0, x} \text { strongly in } L^{2}(\Omega) \text { as } \delta \rightarrow 0 .
\end{gathered}
$$

The parameters $\epsilon>0$ and $\delta>0$ in (3.2) make the problem regular up to the boundary (i.e. uniformly parabolic). The existence of a local in time solution of (3.2) is guaranteed by the classical Schauder estimates 
(see [9]). Now suppose that $u_{\epsilon \delta}$ is a solution of equation (3.2) and that it is continuously differentiable with respect to the time variable and fourth order continuously differentiable with respect to the spatial variable. For the full detailed proof of existence of strong solutions please refer to [16].

Multiplying the equation (3.2) by $\phi(x, t) G_{\epsilon}^{\prime}\left(u_{\epsilon \delta}\right)$, integrating over $\Omega$, and then integrating by parts yield

$$
\begin{aligned}
& \frac{d}{d t} \int_{\Omega} \phi G_{\epsilon}\left(u_{\epsilon \delta}\right) d x- \\
& \int_{\Omega} \phi_{t} G_{\epsilon}\left(u_{\epsilon \delta}\right) d x=\int_{\Omega}\left(1-x^{2}+\delta\right) u_{\epsilon \delta, x}\left[\left(1-x^{2}+\delta\right) u_{\epsilon \delta, x}\right]_{x x} \phi d x+ \\
& \quad \int_{\Omega}\left(1-x^{2}+\delta\right)\left(\left|u_{\epsilon \delta}\right|^{n}+\epsilon\right) G_{\epsilon}^{\prime}\left(u_{\epsilon \delta}\right)\left[\left(1-x^{2}+\delta\right) u_{\epsilon \delta, x}\right]_{x x} \phi_{x} d x= \\
& -\int_{\Omega}\left[\left(1-x^{2}+\delta\right) u_{\epsilon \delta, x}\right]_{x}^{2} \phi d x-\int_{\Omega}\left(1-x^{2}+\delta\right) u_{\epsilon \delta, x}\left[\left(1-x^{2}+\delta\right) u_{\epsilon \delta, x}\right]_{x} \phi_{x} d x- \\
& \quad \int_{\Omega}\left[\left(1-x^{2}+\delta\right)\left(\left|u_{\epsilon \delta}\right|^{n}+\epsilon\right) G_{\epsilon}^{\prime}\left(u_{\epsilon \delta}\right) \phi_{x}\right]_{x}\left[\left(1-x^{2}+\delta\right) u_{\epsilon \delta, x}\right]_{x} d x= \\
& \quad \int_{\Omega}\left[\left(1-x^{2}+\delta\right) u_{\epsilon \delta, x}\right]_{x}^{2} \phi d x+\frac{1}{2} \int_{\Omega}\left[\left(1-x^{2}+\delta\right) u_{\epsilon \delta, x}\right]^{2} \phi_{x x} d x- \\
& \quad \int_{\Omega}\left[\left(1-x^{2}+\delta\right) u_{\epsilon \delta, x}\right]_{x}\left(\left|u_{\epsilon \delta}\right|^{n}+\epsilon\right) G_{\epsilon}^{\prime}\left(u_{\epsilon \delta}\right)\left(\left(1-x^{2}+\delta\right) \phi_{x}\right)_{x} d x- \\
& \quad \int_{\Omega}\left[\left(1-x^{2}+\delta\right) u_{\epsilon \delta, x}\right]_{x}\left(1-x^{2}+\delta\right)\left[\left(\left|u_{\epsilon \delta}\right|^{n}+\epsilon\right) G_{\epsilon}^{\prime}\left(u_{\epsilon \delta}\right)\right]_{u}^{\prime} u_{\epsilon \delta, x} \phi_{x} d x .
\end{aligned}
$$

Integrating (3.8) in time and taking the regularizing parameter $\epsilon \rightarrow 0$, 
by applying the Young inequality and $z^{n} G_{0}^{\prime}(z)=\frac{1}{1-n} z$, we finally get

$$
\begin{gathered}
\int_{\Omega} \phi G_{0}\left(u_{\delta}\right) d x-\iint_{Q_{T}} \phi_{t} G_{0}\left(u_{\delta}\right) d x d t+\iint_{Q_{T}}\left[\left(1-x^{2}+\delta\right) u_{\delta, x}\right]_{x}^{2} \phi d x d t \leqslant \\
\int_{\Omega} \phi G_{0}\left(u_{0, \delta}\right) d x+\frac{1}{2} \iint_{Q_{T}}\left[\left(1-x^{2}+\delta\right) u_{\delta, x}\right]^{2} \phi_{x x} d x d t- \\
\frac{1}{1-n} \iint_{Q_{T}}\left[\left(1-x^{2}+\delta\right) u_{\delta, x}\right]_{x} u_{\delta}\left(\left(1-x^{2}+\delta\right) \phi_{x}\right)_{x} d x d t- \\
\frac{1}{1-n} \iint_{Q_{T}}\left[\left(1-x^{2}+\delta\right) u_{\delta, x}\right]_{x}\left(1-x^{2}+\delta\right) u_{\delta, x} \phi_{x} d x d t \leqslant \\
\quad \int_{\Omega} \phi G_{0}\left(u_{0, \delta}\right) d x+\mu \iint_{Q_{T}}\left[\left(1-x^{2}+\delta\right) u_{\delta, x}\right]_{x}^{2} \phi d x d t+ \\
\frac{2-n}{2(1-n)} \iint_{Q_{T}}\left[\left(1-x^{2}+\delta\right) u_{\delta, x}\right]^{2} \phi_{x x} d x d t+\frac{1}{4 \mu(1-n)^{2}} \iint_{Q_{T}} u_{\delta}^{2} \frac{\left(\left(1-x^{2}+\delta\right) \phi_{x}\right)_{x}^{2}}{\phi} d x d t
\end{gathered}
$$

where $\mu>0$. Choosing $\mu$ in (3.9) such that $0<\mu<1$, we arrive at

$$
\begin{aligned}
& \int_{\Omega} \phi G_{0}\left(u_{\delta}\right) d x- \\
& \quad \iint_{Q_{T}} \phi_{t} G_{0}\left(u_{\delta}\right) d x d t+C \iint_{Q_{T}}\left[\left(1-x^{2}+\delta\right) u_{\delta, x}\right]_{x}^{2} \phi d x d t \leqslant \\
& C \iint_{Q_{T}}\left[\left(1-x^{2}+\delta\right) u_{\delta, x}\right]^{2}\left|\phi_{x x}\right| d x d t+C \iint_{Q_{T}} u_{\delta}^{2} \frac{\left(\left(1-x^{2}+\delta\right) \phi_{x}\right)_{x}^{2}}{\phi} d x d t .
\end{aligned}
$$


Letting $\delta \rightarrow 0$ in (3.10), we deduce that

$$
\begin{gathered}
\int_{\Omega} \phi(T) G_{0}(u) d x-\iint_{Q_{T}} \phi_{t} G_{0}(u) d x d t+ \\
C \iint_{Q_{T}}\left[\left(1-x^{2}\right) u_{x}\right]_{x}^{2} \phi d x d t \leqslant \int_{\Omega} \phi(0) G_{0}\left(u_{0}\right) d x \\
C \iint_{Q_{T}}\left[\left(1-x^{2}\right) u_{x}\right]^{2}\left|\phi_{x x}\right| d x d t+C \iint_{Q_{T}} u^{2} \frac{\left(\left(1-x^{2}\right) \phi_{x}\right)_{x}^{2}}{\phi} d x d t .
\end{gathered}
$$

Taking $\phi(x, t)=\left(1-x^{2}\right)^{\nu} \zeta^{4}(x, t)$ in (3.11) for $\nu>1$, we have

$$
\begin{gathered}
\int_{\Omega}\left(1-x^{2}\right)^{\nu} \zeta^{4}(T) G_{0}(u) d x-\iint_{Q_{T}}\left(1-x^{2}\right)^{\nu}\left(\zeta^{4}\right)_{t} G_{0}(u) d x d t+ \\
C \iint_{Q_{T}}\left(1-x^{2}\right)^{\nu}\left[\left(1-x^{2}\right) u_{x}\right]_{x}^{2} \zeta^{4} d x d t \leqslant \int_{\Omega}\left(1-x^{2}\right)^{\nu} \zeta^{4}(0) G_{0}\left(u_{0}\right) d x+ \\
C \iint_{Q_{T}}\left[\left(1-x^{2}\right) u_{x}\right]^{2}\left[\left(1-x^{2}\right)^{\nu-2} \zeta^{4}+\left(1-x^{2}\right)^{\nu}\left(\zeta^{2} \zeta_{x}^{2}+\zeta^{3}\left|\zeta_{x x}\right|\right)\right] d x d t+ \\
C \int_{Q_{T}} u^{2}\left[\left(1-x^{2}\right)^{\nu-2} \zeta^{4}+\left(1-x^{2}\right)^{\nu+2} \zeta_{x}^{4}+\left(1-x^{2}\right)^{\nu+2} \zeta^{2} \zeta_{x x}^{2}\right] d x d t \leqslant \\
\int_{\Omega}\left(1-x^{2}\right)^{\nu} \zeta^{4}(0) G_{0}\left(u_{0}\right) d x+C \iint_{Q_{T}}\left(1-x^{2}\right)^{\nu} u_{x}^{2}\left[\zeta^{4}+\zeta^{2} \zeta_{x}^{2}+\zeta^{3}|\zeta x x|\right] d x d t+ \\
C \iint_{Q_{T}}\left(1-x^{2}\right)^{\nu-2} u^{2}\left[\zeta^{4}+\zeta_{x}^{4}+\zeta^{2} \zeta_{x x}^{2}\right] d x d t, \quad(3.12)
\end{gathered}
$$

whence we deduce (3.1). 


\subsection{Finite speed of propagation}

For an arbitrary $s>0$ and $0<\delta \leqslant s$ we consider the families of sets

$$
\begin{gathered}
\Omega(s):=\{x \in \bar{\Omega}:|x| \leqslant s\}, Q_{T}(s)=(0, T) \times \Omega(s), \\
K_{T}(s, \delta)=Q_{T}(s) \backslash Q_{T}(s-\delta) .
\end{gathered}
$$

We introduce a nonnegative cutoff function $\eta(\tau)$ from the space $C^{2}\left(\mathbb{R}^{1}\right)$ with the following properties:

$$
\eta(\tau)=\left\{\begin{aligned}
1 & \text { if } \tau \leqslant 0 \\
-\tau^{3}\left(6 \tau^{2}-15 \tau+10\right)+1 & \text { if } 0<\tau<1 \\
0 & \text { if } \tau \geqslant 1
\end{aligned}\right.
$$

Next we introduce our main cut-off functions $\eta_{s, \delta}(x) \in C^{2}(\bar{\Omega})$ such that $0 \leqslant \eta_{s, \delta}(x) \leqslant 1 \forall x \in \bar{\Omega}$ and possess the following properties:

$$
\begin{gathered}
\eta_{s, \delta}(x)=\eta\left(\frac{|x|-(s-\delta)}{\delta}\right)=\left\{\begin{array}{l}
1, x \in \Omega(s-\delta), \\
0, x \in \Omega \backslash \Omega(s),
\end{array}\right. \\
\left|\left(\eta_{s, \delta}\right)_{x}\right| \leqslant \frac{15}{8 \delta},\left|\left(\eta_{s, \delta}\right)_{x x}\right| \leqslant \frac{5(\sqrt{3}-1)}{\delta^{2}}
\end{gathered}
$$

for all $s>0$ and $0<\delta \leqslant s$. Choosing $\zeta^{4}(x, t)=\eta_{s, \delta}(x) e^{-\frac{t}{T}}$ in (3.1), we arrive at

$$
\begin{gathered}
\int_{\Omega(s-\delta)}\left(1-x^{2}\right)^{\nu} u^{2-n}(T) d x+\frac{C}{T} \iint_{Q_{T}(s-\delta)}\left(1-x^{2}\right)^{\nu} u^{2-n} d x d t+ \\
C \iint_{Q_{T}(s-\delta)}\left(1-x^{2}\right)^{\nu+2} u_{x x}^{2} d x d t \leqslant e \iint_{\Omega(s)}\left(1-x^{2}\right)^{\nu} u_{0}^{2-n}(x) d x+ \\
\quad \frac{C}{\delta^{2}} \iint_{K_{T}(s, \delta)}\left(1-x^{2}\right)^{\nu} u_{x}^{2} d x d t+\frac{C}{\delta^{4}} \iint_{K_{T}(s, \delta)}\left(1-x^{2}\right)^{\nu-2} u^{2} d x d t
\end{gathered}
$$


for all $0<\delta \leqslant s$. By (3.16) we deduce that

$$
\begin{gathered}
\left(1-(s-\delta)^{2}\right)^{\nu} \int_{\Omega(s-\delta)} u^{2-n}(T) d x+\frac{C\left(1-(s-\delta)^{2}\right)^{\nu}}{T} \iint_{Q_{T}(s-\delta)} u^{2-n} d x d t+ \\
C\left(1-(s-\delta)^{2}\right)^{\nu} \iint_{Q_{T}(s-\delta)}\left(1-x^{2}\right)^{2} u_{x x}^{2} d x d t \leqslant \frac{C\left(1-(s-\delta)^{2}\right)^{\nu}}{\delta^{2}} \iint_{K_{T}(s, \delta)} u_{x}^{2} d x d t+ \\
\frac{C\left(1-(s-\delta)^{2}\right)^{\nu}}{\delta^{4}} \iint_{K_{T}(s, \delta)}\left(1-x^{2}\right)^{-2} u^{2} d x d t
\end{gathered}
$$

whence

$$
\begin{gathered}
\int_{\Omega(s-\delta)} u^{2-n}(T) d x+\frac{C}{T} \iint_{Q_{T}(s-\delta)} u^{2-n} d x d t+ \\
C\left(1-r_{0}^{2}\right)^{2} \iint_{Q_{T}(s-\delta)} u_{x x}^{2} d x d t \leqslant \frac{C}{\delta^{2}} \iint_{K_{T}(s, \delta)} u_{x}^{2} d x d t+ \\
\frac{C\left(1-r_{0}^{2}\right)^{-2}}{\delta^{4}} \iint_{K_{T}(s, \delta)} u^{2} d x d t=: R(s)
\end{gathered}
$$

for all $0<\delta \leqslant s \leqslant r_{0}$. We apply Lemma A.1 in the region $\Omega(s-\delta)$ to a function $v:=u$ with $a=d=j=2, b=2-n, k=0$ (or $k=1$ ), $N=1$, and $\theta_{1}=\frac{n}{8-3 n}$ (or $\theta_{2}=\frac{4-n}{8-3 n}$ ). Integrating the resulted inequalities with respect to time and taking into account (3.17), we arrive at the following relations:

$$
\begin{aligned}
& A(s-\delta) \leqslant C\left(1-r_{0}^{2}\right)^{-\alpha_{1}} T^{\beta_{1}}(R(s))^{1+\kappa_{1}}+C T(R(s))^{1+\kappa_{3}}, \\
& B(s-\delta) \leqslant C\left(1-r_{0}^{2}\right)^{-\alpha_{2}} T^{\beta_{2}}(R(s))^{1+\kappa_{2}}+C T(R(s))^{1+\kappa_{3}},
\end{aligned}
$$

where

$$
\begin{gathered}
A(s):=\iint_{Q_{T}(s)} u^{2} d x d t, B(s):=\iint_{Q_{T}(s-\delta)} u_{x}^{2} d x d t, \\
\alpha_{1}=\frac{4(n+4)}{8-3 n}, \alpha_{2}=\frac{4(6-n)}{8-3 n}, \beta_{1}=\frac{4(2-n)}{8-3 n}, \beta_{2}=\frac{2(2-n)}{8-3 n},
\end{gathered}
$$




$$
\kappa_{1}=\frac{4 n}{8-3 n}, \kappa_{2}=\frac{2 n}{8-3 n}, \kappa_{3}=\frac{n}{2-n} .
$$

Since all integrals on the right-hand sides of (3.18), (3.19) vanish as $T \rightarrow 0$ and $u \in L^{2}\left(0, T ; H^{1}\left(-r_{0}, r_{0}\right)\right)$, then for sufficiently small $T$ we get

$$
\begin{aligned}
& A(s-\delta) \leqslant C_{3}\left(1-r_{0}^{2}\right)^{-\alpha_{1}} T^{\beta_{1}}\left(\delta^{-4} A(s)+\delta^{-2} B(s)\right)^{1+\kappa_{1}} \\
& B(s-\delta) \leqslant C_{4}\left(1-r_{0}^{2}\right)^{-\alpha_{2}} T^{\beta_{2}}\left(\delta^{-4} A(s)+\delta^{-2} B(s)\right)^{1+\kappa_{2}} .
\end{aligned}
$$

Let us denote by

$$
\begin{gathered}
D(s):=A^{1+\kappa_{2}}(s)+B^{1+\kappa_{1}}(s), \kappa=\left(1+\kappa_{1}\right)\left(1+\kappa_{2}\right), \\
C_{5}(T):=2^{\kappa-1} \max \left\{\left[C_{3}\left(1-r_{0}^{2}\right)^{-\alpha_{1}} T^{\beta_{1}}\right]^{1+\kappa_{2}},\left[C_{4}\left(1-r_{0}^{2}\right)^{-\alpha_{2}} T^{\beta_{2}}\right]^{1+\kappa_{1}}\right\} .
\end{gathered}
$$

Without loss of generality, we can define the function

$$
\tilde{D}(s)=D(s) \text { if } s \in\left(0, r_{0}\right] \text {, and } \tilde{D}(s)=0 \text { if } s>r_{0} .
$$

Then by (3.20), (3.21) we arrive at

$$
\tilde{D}(s-\delta) \leqslant C_{5}(T)\left(\delta^{-4 \kappa} \tilde{D}^{1+\kappa_{1}}(s)+\delta^{-2 \kappa} \tilde{D}^{1+\kappa_{2}}(s)\right)
$$

for all $s \in \mathbb{R}^{+}$and $\delta \in\left(0, r_{0}\right]$. Choosing

$$
\delta(s)=\max \left\{\left[4 C_{5}(T) \tilde{D}^{\kappa_{1}}(s)\right]^{\frac{1}{4 \kappa}},\left[4 C_{5}(T) \tilde{D}^{\kappa_{2}}(s)\right]^{\frac{1}{2 \kappa}}\right\}
$$

in (3.22), we find that

$$
\tilde{D}(s-\delta(s)) \leqslant \frac{1}{2} \tilde{D}(s)
$$

whence it follows

$$
\delta(s-\delta(s)) \leqslant \gamma \delta(s) \quad \forall s \in \mathbb{R}^{+},
$$

where $\gamma=\max \left\{2^{-\frac{\kappa_{1}}{4 \kappa}}, 2^{-\frac{\kappa_{2}}{2 \kappa}}\right\}<1$. Applying Stampacchia's lemma (see Lemma A.2) to (3.23), we obtain that

$$
\delta(s)=0 \text { for all } s \leqslant r_{0}-\frac{\delta\left(r_{0}\right)}{1-\gamma} .
$$


Next, we will find the upper bound for $\delta\left(r_{0}\right)$. In view of Theorem 1, $\left(1-x^{2}\right)^{\frac{\nu-2}{2}} u \in L^{2}\left(Q_{T}\right)$ and $\left(1-x^{2}\right)^{\frac{\nu}{2}} u_{x} \in L^{2}\left(Q_{T}\right)$ for any $\nu>1$ then the right-hand side of (3.16) is bounded for all $T>0$. So, taking $s=2 r_{0}$ and $\delta=r_{0}$ in (3.18) and (3.19), we obtain that $\tilde{D}\left(r_{0}\right) \leqslant C_{6} C_{5}(T)$, whence

$$
\delta\left(r_{0}\right) \leqslant C_{7}\left(1-r_{0}^{2}\right)^{-\frac{2(6-n)}{8-3 n}} T^{\frac{2-n}{8-3 n}} .
$$

This implies the upper bound for speed of propagation to solution support, i. e.

$$
\Gamma(T) \leqslant r_{0}-C_{8} T^{\frac{2-n}{8-3 n}} \forall T \leqslant T^{*}:=\left(\frac{r_{0}}{C_{8}}\right)^{\frac{8-3 n}{2-n}}
$$

for any $r_{0} \in(0,1)$, where $C_{8}=\frac{C_{7}}{1-\gamma}\left(1-r_{0}^{2}\right)^{-\frac{2(6-n)}{8-3 n}}$.

\subsection{Exact upper bound for speed of propagation}

In this section we refine the estimate (3.24). Applying Lemma A.1 in the region $\Omega(s) \backslash \Omega(s-\delta)$ to a function $v:=u$ with $a=d=j=2$, $b=1, k=0$ (or $k=1$ ), $N=1$, and $\theta_{1}=\frac{1}{5}$ ( or $\theta_{2}=\frac{3}{5}$ ), and integrating the resulted inequalities with respect to time, taking into account the mass conservation (2.4), we arrive at the following estimates:

$$
\begin{aligned}
& \iint_{K_{T}(s, \delta)} u^{2} d x d t \leqslant C T^{1-\theta_{1}} M^{2\left(1-\theta_{1}\right)}\left(\iint_{K_{T}(s, \delta)} u_{x x}^{2} d x d t\right)^{\theta_{1}}+C \delta^{-1} T M^{2}, \\
& \iint_{K_{T}(s, \delta)} u_{x}^{2} d x d t \leqslant C T^{1-\theta_{2}} M^{2\left(1-\theta_{2}\right)}\left(\iint_{K_{T}(s, \delta)} u_{x x}^{2} d x d t\right)^{\theta_{2}}+C \delta^{-3} T M^{2} .
\end{aligned}
$$

Using (3.25), (3.26) and Young inequality, from (3.17) we find that

$$
\begin{array}{r}
\int_{\Omega(s-\delta)} u^{2-n}(T) d x+\frac{C}{T} \iint_{Q_{T}(s-\delta)} u^{2-n} d x d t+C\left(1-r_{0}^{2}\right)^{2} \iint_{Q_{T}(s-\delta)} u_{x x}^{2} d x d t \leqslant \\
\varepsilon\left(1-r_{0}^{2}\right)^{2} \iint_{K_{T}(s, \delta)} u_{x x}^{2} d x d t+C_{\varepsilon} \delta^{-5}\left(1-r_{0}^{2}\right)^{-3} T M^{2}
\end{array}
$$


where $\varepsilon>0$. Selecting $\varepsilon \in\left(0,2^{-5}\right)$ enough small and making standard iteration process, we get

$$
\begin{aligned}
& \int_{\Omega(s-\delta)} u^{2-n}(T) d x+\frac{C}{T} \iint_{Q_{T}(s-\delta)} u^{2-n} d x d t+ \\
& C\left(1-r_{0}^{2}\right)^{2} \iint_{Q_{T}(s-\delta)} u_{x x}^{2} d x d t \leqslant C \delta^{-5}\left(1-r_{0}^{2}\right)^{-3} T M^{2} .
\end{aligned}
$$

Taking $s=2 \Gamma(T)$ and $\delta=\Gamma(T)$ in (3.27), we obtain that

$$
\iint_{Q_{T}(\Gamma(T))} u_{x x}^{2} d x d t \leqslant C \Gamma^{-5}(T)\left(1-r_{0}^{2}\right)^{-5} T M^{2},
$$

whence, similar to (3.25) and (3.26), we have $A(\Gamma(T)) \leqslant C \Gamma^{-1}(T)\left(1-r_{0}^{2}\right)^{-1} T M^{2}, B(\Gamma(T)) \leqslant C \Gamma^{-3}(T)\left(1-r_{0}^{2}\right)^{-3} T M^{2}$.

Hence,

$$
\begin{gathered}
\delta(\Gamma(T)) \leqslant C \max \left\{\left[\Gamma^{-\kappa_{1}}(T)\left(1-r_{0}^{2}\right)^{-\left(\kappa_{1}+\alpha_{1}\right)} T^{\kappa_{1}+\beta_{1}} M^{2 \kappa_{1}}\right]^{\frac{1}{4\left(1+\kappa_{1}\right)}},\right. \\
\left.\left[\Gamma^{-3 \kappa_{2}}(T)\left(1-r_{0}^{2}\right)^{-\left(3 \kappa_{2}+\alpha_{2}\right)} T^{\kappa_{2}+\beta_{2}} M^{2 \kappa_{2}}\right]^{\frac{1}{2\left(1+\kappa_{2}\right)}}\right\}= \\
C_{9} \max \left\{\Gamma^{-\frac{n}{n+8}}(T) T^{\frac{2}{n+8}}, \Gamma^{-\frac{3 n}{8-n}}(T) T^{\frac{2}{8-n}}\right\} .
\end{gathered}
$$

Thus, we have

$$
\Gamma(T)+C_{10} \max \left\{\Gamma^{-\frac{n}{n+8}}(T) T^{\frac{2}{n+8}}, \Gamma^{-\frac{3 n}{8-n}}(T) T^{\frac{2}{8-n}}\right\} \leqslant r_{0},
$$

where $C_{10}=\frac{C_{9}}{1-\gamma}$. Now we use the following calculus result: let $a>0$ and $b>0$ then the function $f(x)=x+a x^{-b}$ for all $x \geqslant 0$ has minimum at $x_{\min }=(a b)^{\frac{1}{1+b}}$ and $f\left(x_{\min }\right)=\frac{1+b}{b} x_{\min }$. Hence, minimizing the righthand side, we obtain that

$$
\Gamma_{\text {opt }}(T)=C_{0} T^{\frac{1}{n+4}} \quad \forall T \leqslant T^{*} .
$$

This proves Theorem 1 completely. 


\section{Appendix A}

Lemma A.1 ([14]). If $\Omega \subset \mathbb{R}^{N}$ is a bounded domain with piecewisesmooth boundary, $a>1, b \in(0, a), d>1$, and $0 \leqslant k<j, k, j \in \mathbb{N}$, then there exist positive constants $d_{1}$ and $d_{2}\left(d_{2}=0\right.$ if $\Omega$ is unbounded) depending only on $\Omega, d, j, b$, and $N$ such that the following inequality is valid for every $v(x) \in W^{j, d}(\Omega) \cap L^{b}(\Omega)$ :

$\left\|D^{k} v\right\|_{L^{a}(\Omega)} \leqslant d_{1}\left\|D^{j} v\right\|_{L^{d}(\Omega)}^{\theta}\|v\|_{L^{b}(\Omega)}^{1-\theta}+d_{2}\|v\|_{L^{b}(\Omega)}, \theta=\frac{\frac{1}{b}+\frac{k}{N}-\frac{1}{a}}{\frac{1}{b}+\frac{j}{N}-\frac{1}{d}} \in\left[\frac{k}{j}, 1\right)$.

Note that if $\Omega=B(0, R) \backslash B(0, r)$, where $B(0, x)$ is ball with the radius $x$ and the origin at 0 , then $d_{2}=c(R-r)^{-\frac{(a-b) N}{a b}-k}$.

Lemma A.2 ([15]). Assume that $f(s)$ is nonnegative nondecreasing function satisfying the following inequality

$$
f(s-f(s)) \leqslant \varepsilon f(s) \forall s \leqslant s_{0},
$$

where $\varepsilon \in(0,1)$. Then $f(s)=0$ for all $s \leqslant s_{0}-\frac{f\left(s_{0}\right)}{1-\varepsilon}$.

\section{References}

[1] E. Beretta, M. Bertsch, and R. Dal Passo. Nonnegative solutions of a fourth-order nonlinear degenerate parabolic equation, Archive for rational mechanics and analysis, 129(2): 175-200, 1995.

[2] F. Bernis, A. Friedman. Higher order nonlinear degenerate parabolic equations, J. Differential Equations, 83(1): 179-206, 1990.

[3] F. Bernis. Finite speed of propagation and continuity of the interface for thin viscous flows. Adv. Differential Equations, 1(3): 337-368, 1996. 
[4] F. Bernis. Finite speed of propagation for thin viscous flows when $2 \leq n<3$. Comptes Rendus de l'Académie des Sciences. Série I. Mathématique, 322(12): 1169-1174, 1996.

[5] F. Bernis, L.A. Peletier and S. M. Williams. Source type solutions of a fourth order nonlinear degenerate parabolic equation. Nonlinear Anal., 18: 217-234, 1992.

[6] Andrea L. Bertozzi et al. Singularities and similarities in interface flows, Trends and perspectives in applied mathematics. Springer New York, 155-208, 1994.

[7] Marina Chugunova, Mary C. Pugh, and Roman M. Taranets. Nonnegative solutions for a long-wave unstable thin film equation with convection, SIAM Journal on Mathematical Analysis, 42(4): 1826-1853, 2010.

[8] Marina Chugunova, and Roman M. Taranets. Qualitative analysis of coating flows on a rotating horizontal cylinder, International Journal of Differential Equations, 2012: Article ID 570283, 30 pages, 2012.

[9] Avner Friedman. Interior estimates for parabolic systems of partial differential equations, J. Math. Mech., 7(3): 393-417, 1958.

[10] Josephus Hulshof, and Andrey E. Shishkov. The thin film equation with $2 \leq n<3$ : finite speed of propagation in terms of the $L^{1}$ norm. Adv. Differential Equations, 3(5):625-642, 1998.

[11] D. Kang, A. Nadim, and M. Chugunova. Dynamics and equilibria of thin viscous coating films on a rotating sphere, Journal of Fluid Mechanics, 791: 495-518, 2016.

[12] D. Kang, A. Nadim, and M. Chugunova. Marangoni effects on a thin liquid film coating a sphere with axial or radial thermal gradients, Physics of Fluids, 29: 072106-1-072106-15, 2017. 
[13] D. Kang, Tharathep Sangsawang and Jialun Zhang. Weak solution of a doubly degenerate parabolic equation, arXiv:1610.06303v2, 2017.

[14] L. Nirenberg. An extended interpolation inequality. Ann. Scuola Norm. Sup. Pisa (3), 20: 733-737, 1966.

[15] A. E. Shishkov. Dynamics of the geometry of the support of the generalized solution of a higher-order quasilinear parabolic equation in divergence form, Differ. Uravn., 29(3): 537-547, 1993.

[16] Roman M. Taranets. Strong solutions of the thin film equation in spherical geometry. arXiv:1709.10496, 2017.

[17] D. Takagi, and Herbert E. Huppert. Flow and instability of thin films on a cylinder and sphere, Journal of Fluid Mechanics, 647: 221-238, 2010.

[18] S.K. Wilson. The onset of steady Marangoni convection in a spherical geometry, Journal of Engineering Mathematics, 28: 427-445, 1994. 